\title{
Las abejas del género Agapostemon (Hymenoptera: Halictidae) del estado de Nuevo León, México
}

\section{Bees of the genus Agapostemon (Hymenoptera: Halictidae) of the state of Nuevo León, Mexico}

\author{
Liliana Ramírez-Freire ${ }^{1}$, Glafiro José Alanís-Flores ${ }^{1}$, Ricardo Ayala-Barajas², Humberto Quiroz -Martínez ${ }^{1}$ \\ y Carlos GerardoVelazco-Macías ${ }^{3}$
}

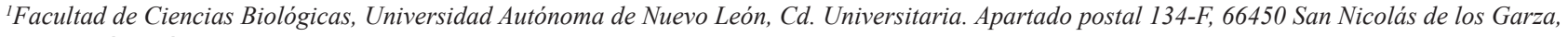
Nuevo León, México.

${ }^{2}$ Estación de Biología Chamela (Sede Colima) Instituto de Biología, Universidad Nacional Autónoma de México. Apartado postal 21, 48980 San Patricio, Jalisco, México.

${ }^{3}$ Parques y Vida Silvestre. Av. Alfonso Reyes norte s/n, interior del Parque Niños Héroes, lateral izquierda, acceso 3, 64290 Monterrey, Nuevo León, México.

\biolily@gmail.com

\begin{abstract}
Resumen. Se realizó un estudio faunístico de las abejas del género Agapostemon (Halictidae) en el estado de Nuevo León, México para conocer las especies presentes, su distribución, relación con la flora y tipos de vegetación del estado. La metodología se basó en la revisión de literatura y de bases de datos de colecciones entomológicas, y en muestreos en campo donde se utilizó red entomológica y platos trampa de colores amarillo, azul, rosa (tonos fluorescentes) y blanco. Sólo en 20 de los 35 muestreos que se realizaron se obtuvieron ejemplares del género. Se recolectaron 11 especies, 2 de las cuales son registros nuevos para el estado (A. nasutus y A. splendens). El 12.31\% de los ejemplares se obtuvo mediante el uso de red y el $87.84 \%$ con los platos trampa; el color amarillo fue el preferido por las abejas. Las especies con mayor distribución fueron A. tyleri y A. angelicus /A. texanus; se recolectaron en 9 especies de plantas pertenecientes a 6 familias. Helianthus annuus (Compositae) presentó la mayor diversidad con 4 especies de Agapostemon. Los muestreos se realizaron en 10 diferentes tipos de vegetación y la mayor riqueza de especies se obtuvo en diversos matorrales y en la vegetación de disturbio.
\end{abstract}

Palabras clave: abejas nativas, Apoidea, polinizadores, abejas del sudor, matorrales, platos trampa.

\begin{abstract}
A study of the bee genus Agapostemon (Halictidae) in the state of Nuevo León, Mexico was carried out to learn about species numbers, distribution, relationship to flora and vegetation types of the state. Methodology was based on a review of literature and databases from enthomological collections, for field sampling net and pan traps were used in yellow, blue, pink (fluorescent colors) and white. A total of 35 sites along the state were visited, Agapostemon specimens were found only in 20 sites. We collected 11 species, 2 of them as new records for the state (A. nasutus and A. splendens). From the total specimens, $12.31 \%$ was obtained with the use of net and $87.84 \%$ using pan traps, being yellow the preferred color. The most widely distributed species were A. tyleri and A. angelicus/A. texanus. Specimens were collected from 9 plants species belonging to 6 families, Helianthus annuus (Compositae) showed the highest species diversity with 4 Agapostemon. Ten different types of vegetation which were sampled, being different brush types and perturbed vegetation the ones with higher species richness.
\end{abstract}

Key words: native bees, Apoidea, pollinators, sweat bees, bushes, pan traps.

\section{Introducción}

La relación que guardan las abejas silvestres con las angiospermas ocupa una posición clave en el mantenimiento y conservación de los ecosistemas naturales y agrícolas (Dafni y O'Toole, 1994; Buchmann y Nabhan, 1996; Daily, 1997). Dado que los servicios ecológicos que brindan las

Recibido: 01 abril 2011; aceptado: 21 junio 2011 abejas nativas son importantes, es necesario continuar los inventarios de estos organismos (O'Toole, 1993). Hay que considerar la alarma internacional que se ha suscitado debido a la disminución de las poblaciones de abejas, fenómeno que primero se detectó en Apis mellifera; sin embargo, las especies de abejas nativas no están exentas de ese riesgo, ya que los principales causantes son el uso de agroquímicos, los monocultivos y la deforestación (Dias et al., 1999).

La riqueza de abejas conocidas de México se estima en 1589 especies distribuidas en 153 géneros y 6 fami- 
lias, las que ordenadas de mayor a menor, según el número de especies, son: Apidae, Andrenidae, Megachilidae, Halictidae, Colletidae y Melittidae (Ayala et al., 1998; Michener, 2000). México es el país que tiene la mayor cantidad de géneros de Halictidae, que constituye un componente importante de las abejas de regiones tropicales y templadas (Ayala et al., 1998). Uno de sus géneros es Agapostemon, el cual incluye especies tanto de amplia distribución como endémicas estrictas; se distribuye desde el sur de Canadá hasta América del Sur. Se diferencia del resto de los miembros de Halictidae por la presencia de una carina que rodea totalmente la superficie posterior del propodeo y por la coloración metálica de cabeza y tórax que contrasta con la no metálica del abdomen en la mayoría de las hembras y casi todos los machos. Además, las hembras se distinguen por los 3-4 (a veces 7) dientes grandes espatulados en el espolón tibial de las patas traseras y por la larga carina paralela contigua que se extiende posterodorsalmente del margen anteroventral de la gena, mientras que los machos pueden diferenciarse por la fusión de los primeros 2 tarsómeros de la pata posterior y por el ensanchamiento del fémur y en menor medida de las tibias de las patas posteriores de muchas de las especies (Roberts, 1972).

Para México se han registrado 15 especies de $A g a-$ postemon en 27 estados, de las cuales $A$. nasutus, $A$, texanus y A. leunculus tienen la distribución más amplia; estan presentes en 20, 17 y 15 estados, respectivamente. Sonora, con 7 especies, ocupa el primer lugar en diversidad de especies; el segundo, Baja California Norte y Chihuahua con 6, y el tercero Chiapas, Durango, Jalisco, Nuevo León, Puebla, San Luis Potosí y Veracruz con 5 (Ayala et al., 1996; Ascher y Pickering, 2010; UNIBIO, 2011).

Para Nuevo León, Ayala et al.(1996) registran $A$. melliventris, A. texanus y A. tyleri; UNIBIO (2011); en la base de datos de Abejas de México de la Colección Nacional de Insectos (CNIN) sólo se registra $A$. texanus; la Colección Himenopterológica del Museo de Zoología Alfonso L. Herrera (Yáñez-Ordóñez e Hinojosa-Díaz, 2004) no tiene ningún registro; las bases de datos del American Museum of Natural History registran A. leunculus, A. melliventris, A. obliquus y A. texanus, y en Snow Entomology Collection,University of Kansas Database está el registro de A. tyleri (Ascher y Pickering, 2010).

El objetivo del trabajo fue hacer un inventario de las especies del género Agapostemon en Nuevo León, conocer las plantas y tipos de vegetación en los que se pueden encontrar y reunir material biológico para formar una colección en el estado.

\section{Materiales y métodos}

Área de estudio. El estado de Nuevo León se ubica en el noreste de la República Mexicana (Fig. 1), tiene una superficie de $64081.94 \mathrm{~km}^{2}$, con elevaciones de 200 a $3700 \mathrm{~m}$. Está dividido en 3 regiones fisiográficas: la Sierra Madre Oriental, la Gran Llanura Norteamericana y la Planicie Costera del Golfo (Alanís-Flores et al., 1996). Predominan los climas semisecos, extremosos y precipitación pluvial variable, con promedio anual de 300-600 $\mathrm{mm}$. Los climas secos y semisecos se presentan principalmente en la región norte oriental y sur occidental, ambas áreas separadas por la sierra Madre Oriental, siendo ésta donde se presentan climas semicálidos, templados y semifríos en la zona centro y sur (INEGI, 1986). La vegetación se compone principalmente de matorrales desérticos y semidesérticos de diversa composición florística, en la región de la Sierra Madre Oriental se observan bosques con asociaciones de encinos, pinos y coníferas. En menor grado se observan sucesiones edafológicas como vegetación gipsófila o vegetación halófita (Treviño-Garza et al., 2001).

Métodos. Se reunió literatura taxonómica sobre Agapostemon (Mitchell, 1960; Roberts, 1972; Michener, 2000; Janjic y Packer, 2003; Ascher y Pickering, 2010). Se consultaron las bases de datos de la Colección Himenopterológica del Museo de Zoología Alfonso L. Herrera, Facultad de Ciencias, UNAM; la de la Colección Nacional de Insectos (CNIN) de México; la Bee Species Database, American Museum of Natural History y la Snow Entomology Collection, University of Kansas Natural History Museum (las 2 últimas en Ascher y Pickering, 2010).

Para el trabajo de campo se realizaron 35 muestreos dentro del estado entre febrero del 2009 y mayo del 2010. Para la captura de ejemplares se utilizó red entomológica sobre plantas en floración por periodos de 30 minutos por planta y platos trampa de colores amarillo, azul, rosa (en tonalidades fluorescentes) y blanco, conteniendo solución jabonosa (agua de grifo con detergente líquido para trastes). En cada sitio de muestreo fueron colocadas 30 trampas de cada color, en un área de $1000 \mathrm{~m}^{2}$, dejándose en el sitio de 8:00 a 18:00 h (Droege, 2008). Las abejas obtenidas con red entomológica se montaron el mismo día de recolecta. Las recolectadas en platos trampa se procesaron mediante la técnica de Droege (2008). La identificación de los ejemplares se basó en Roberts (1972), Michener et al. (1994) y Ascher y Pickering (2010). Junto con los ejemplares de abejas se recolectaron muestras botánicas de las plantas visitadas, las cuales se herborizaron para su posterior identificación. Para definir los tipos de vegetación donde fueron realizados los muestreos se utilizó el criterio 
de INEGI (1986). Los ejemplares serán depositados en la Colección Entomológica de la Faculta de Ciencias Biológicas de la Universidad Autónoma de Nuevo León.

\section{Resultados}

De los 35 muestreos realizados en este estudio, sólo en 20 se obtuvieron ejemplares de Agapostemon (Apéndice 1, Fig. 1) aportando nuevos registros de su distribución. Se recolectaron 236 ejemplares (225 hembras y 11 machos), y se identificaron 11 especies. El detalle de éstas es el siguiente: A. melliventris (14 + ), A. obliquus (11 + ), A. texanus $(2 \hat{O})$, A. tyleri ( 81 q, 4 đ). Se registran por primera vez para Nuevo León $A$. nasutus $(1 \stackrel{+}{ }, 1$ đ) y $A$. splendens $\left(\begin{array}{l}1 \\ \bigcirc\end{array}\right)$, y un grupo de hembras (111) que no fue posible diferenciar entre $A$. angelicus y $A$. texanus, por lo que en este trabajo se indican como A. angelicus / $A$. texanus. De los organismos restantes, las hembras se designaron con número y con letra los machos: Agapostemon sp. 1 (6 +), Agapostemon sp. 2 (1 ㅇ), Agapostemon sp. A


registrada por Ascher y Pickering (2010), no se recolectó pero está registrada para la localidad de Chipinque, por lo que se incluyó en este trabajo. Las 2 especies más abundantes fueron $A$. angelicus / A. texanus (47\%) y A. tyleri (36\%), que representan el $83 \%$ del material recolectado.

Del muestreo realizado con red se recolectó el $12.31 \%$ de ejemplares en 9 especies de plantas: Ehretia anacua (Boraginaceae), Eysenhardtia texana (Fabaceae), Leucophyllum frutescens (Scrophulariaceae), Ludwigia octovalis
(Onagraceae), Opuntia microdasys y Opuntia sp. (Cactaceae), y Helianthus annuus, Parthenium argentatum y una especie no identificada (Compositae) (véase Apéndice1). La mayor diversidad de abejas, se presentó en $H$. annuus, con 4 especies recolectadas, seguida por $L$. frutescens con 2; el resto de las plantas sólo presentó 1. Agapostemon splendens fue la única especie que se recolectó exclusivamente con red sobre las plantas (Cuadro 1).

El $87.89 \%$ de los ejemplares se recolectó con los platos trampa. Se observó preferencia por el color amarillo (49.13\%), seguido del blanco y del azul, con el $23.31 \%$ y el $15.25 \%$ respectivamente, mientras que ningún ejemplar fue atraído por el rosa. Agapostemon sp. A, Agapostemon sp. 1 y Agapostemon sp. 2 se obtuvieron solamente bajo este método. Abejas como Agapostemon sp. A, Agapostemon sp. B y Agapostemon sp. 2 se recolectaron exclusivamente en el color amarillo y A. angelicus / A. texanus, A. obliquus y $A$. tyleri tuvieron una mayor frecuencia en este color, aunque también se presentaron en el azul y blanco. A. nasutus, y Agapostemon sp. B fueron recolectadas con la misma frecuencia tanto en red como en platos trampa (Cuadro 1).

Por lo que respecta a la distribución de las especies, se encontró que las abejas que tienen un rango más amplio en el estado son: A. tyleri en 12 localidades (Fig. 2) con 8 de los 10 tipos de vegetación y $A$. angelicus $/ A$. texanus en 8 sitios (Fig. 3) con 5 tipos de vegetación; por el contrario, las especies localizadas en un solo lugar fueron: A. splendens, Agapostemon sp. 1, Agapostemon sp. 2, Agapostemon sp. A y Agapostemon sp. B (Figs. 4, 5). La distribución de

Cuadro 1. Proporción de abejas recolectadas de acuerdo con su especie y el método de captura (red entomológica o plato trampa)

\begin{tabular}{|c|c|c|c|c|c|c|c|}
\hline \multirow[t]{2}{*}{ Especie } & \multirow[t]{2}{*}{ Proporción* } & \multirow{2}{*}{$\begin{array}{c}\text { Red } \\
\text { entomológica }\end{array}$} & \multicolumn{5}{|c|}{ Platos trampa } \\
\hline & & & & Amarillo & Azul & Blanco & Rosa \\
\hline A. angelicus/A.texanus & 47 & 1.3 & 45.76 & 28.39 & 6.36 & 11.02 & 0 \\
\hline A. leunculus & 0 & 0 & 0 & 0 & 0 & 0 & 0 \\
\hline A. melliventris & 5.9 & 4.66 & 1.27 & 0 & 0.42 & 0.85 & 0 \\
\hline A. nasutus & 0.8 & 0.42 & 0.42 & 0.42 & 0 & 0 & 0 \\
\hline A. obliquus & 4.7 & 1.7 & 2.97 & 1.69 & 1.27 & 0 & 0 \\
\hline A. splendens & 0.4 & 0.42 & 0 & 0 & 0 & 0 & 0 \\
\hline A. texanus & 0.8 & 0.42 & 0.42 & 0.42 & 0 & 0 & 0 \\
\hline A. tyleri & 36 & 2.97 & 33.05 & 16.1 & 5.93 & 11.02 & 0 \\
\hline Agapostemon sp. A & 0.4 & 0 & 0.42 & 0.42 & 0 & 0 & 0 \\
\hline Agapostemon sp. B & 0.8 & 0.42 & 0.42 & 0.42 & 0 & 0 & 0 \\
\hline Agapostemon sp. 1 & 2.5 & 0 & 2.54 & 0.85 & 1.27 & 0.42 & 0 \\
\hline \multirow[t]{2}{*}{ Agapostemon sp. 2} & 0.4 & 0 & 0.42 & 0.42 & 0 & 0 & 0 \\
\hline & $100 \%$ & $12.31 \%$ & $87.69 \%$ & $49.13 \%$ & $15.25 \%$ & $23.31 \%$ & $0 \%$ \\
\hline
\end{tabular}

* Para los porcentajes de abejas recolectadas por los 2 métodos, así como para el de los colores de los platos trampa, las proporciones se basan en el total de ejemplares recolectados. 
Cuadro 2. Tipos de vegetación presentes en los muestreos y especies de abejas del género Agapostemon compartidas

\begin{tabular}{|c|c|c|c|c|c|c|c|c|c|c|c|c|}
\hline 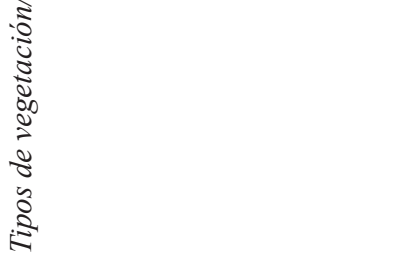 & 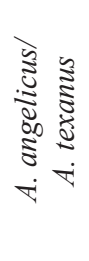 & 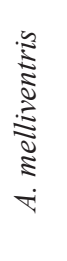 & 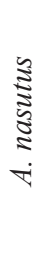 & 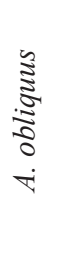 & $\begin{array}{l}\frac{2}{5} \\
\frac{5}{5} \\
\frac{5}{3} \\
\frac{\pi}{2} \\
\dot{\pi}\end{array}$ & 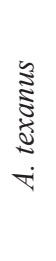 & $\begin{array}{l}\frac{\pi}{3} \\
\frac{\pi}{2} \\
\dot{\pi}\end{array}$ & $\begin{array}{l}\varangle \\
\dot{\infty} \\
\dot{\nabla}\end{array}$ & $\begin{array}{l}m \\
\text { की } \\
\dot{\nabla}\end{array}$ & $\begin{array}{l}\vec{D} \\
\text { क } \\
\dot{\nabla}\end{array}$ & $\begin{array}{l}\text { N } \\
\text { क } \\
\dot{\nabla}\end{array}$ & 吾 \\
\hline Bosque de pino & & & & & & & 3 & & & & & 3 \\
\hline Ecotono* & 1 & & & & & & 4 & & & & & 5 \\
\hline Matorral bajo subinerme & & 1 & & & & & 1 & 1 & & 6 & & 9 \\
\hline Matorral de gobernadora & 59 & & & & & & 37 & & & & 1 & 97 \\
\hline Matorral desértico micrófilo & 4 & & & 2 & & & 29 & & & & & 35 \\
\hline Matorral espinoso tamaulpeco & 41 & & & & & & & & & & & 41 \\
\hline Matorral mediano espinoso & & & & & & 1 & 1 & & & & & 2 \\
\hline Matorral submontano & & & & & & & 4 & & & & & 4 \\
\hline Vegetación riparia & & & 1 & & & & & & & & & 1 \\
\hline Vegetación de disturbio & 6 & 13 & 1 & 9 & 1 & 1 & 6 & & 2 & & & 39 \\
\hline Total & 111 & 14 & 2 & 11 & 1 & 2 & 85 & 1 & 2 & 6 & 1 & 236 \\
\hline
\end{tabular}

*Zona de transición entre matorral submontano y bosque de pino.

Nota: Agapostemon leunculus no se incluyó en el cuadro por no haber sido recolectada durante esta investigación y la información disponible de la localidad no es suficiente para definir con exactitud el tipo de vegetación en la que fue encontrada.

A. obliquus, así como de $A$. melliventris y $A$. nasutus, se puede observar en las figuras 4 y 6 , respectivamente.

Los tipos de vegetación presentes en los sitios de recolecta son 10 (Cuadro 2). En los distintos matorrales (principalmente el matorral bajo subinerme) y en la vegetación de disturbio fue donde se obtuvo una mayor riqueza de especies; el mayor número de ejemplares se recolectó en el matorral de gobernadora (Larrea tridentata).

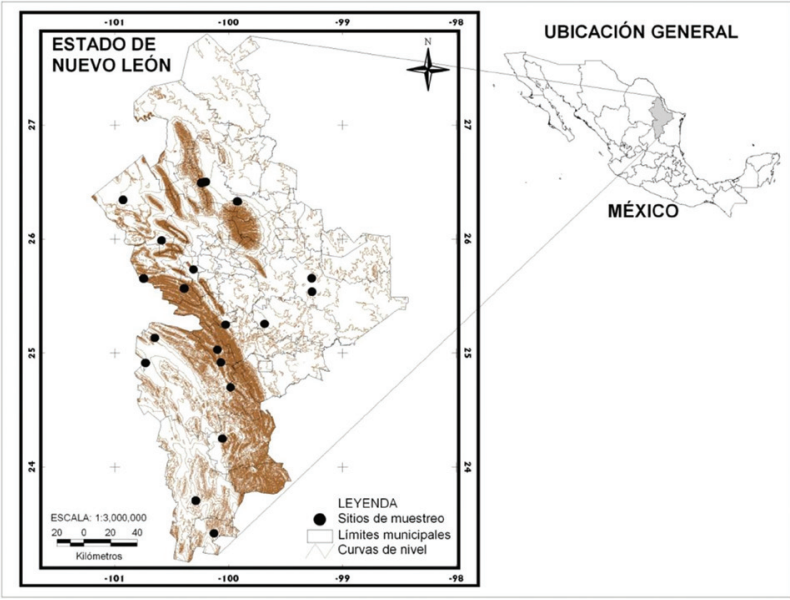

Figura 1. Área de estudio que señala los sitios donde fue recolectado el género Agapostemon.

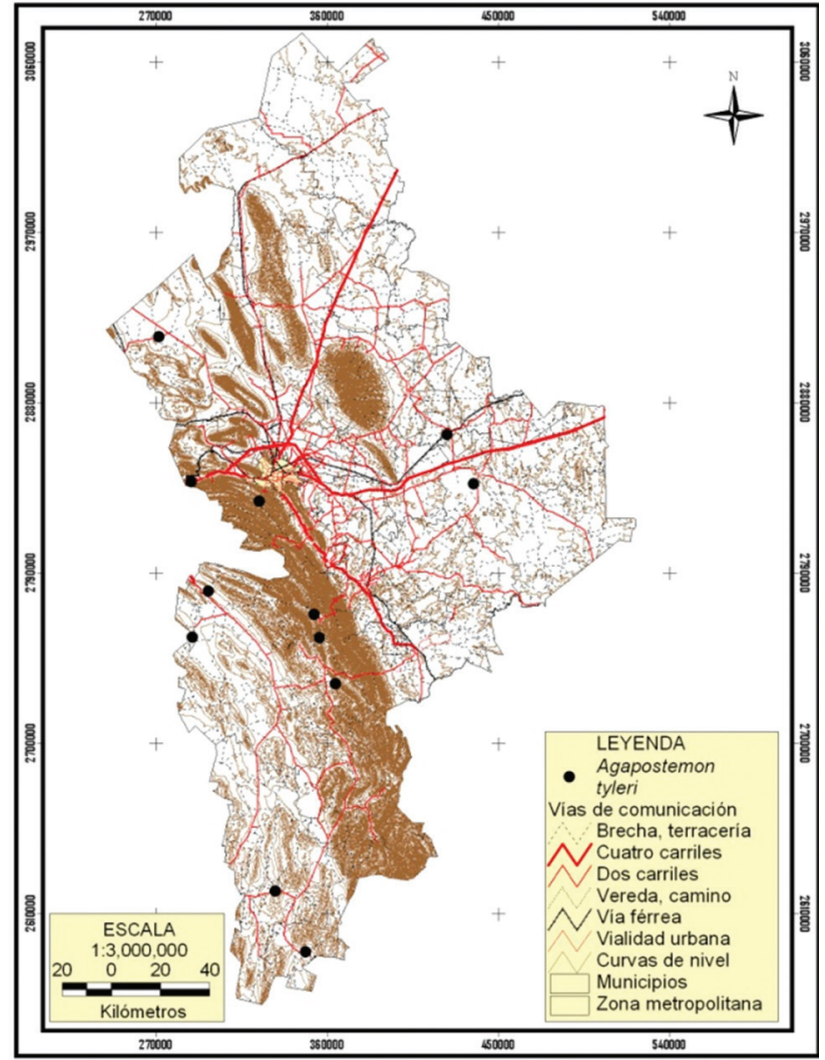

Figura 2. Distribución de Agapostemon tyleri en el estado de Nuevo León. 


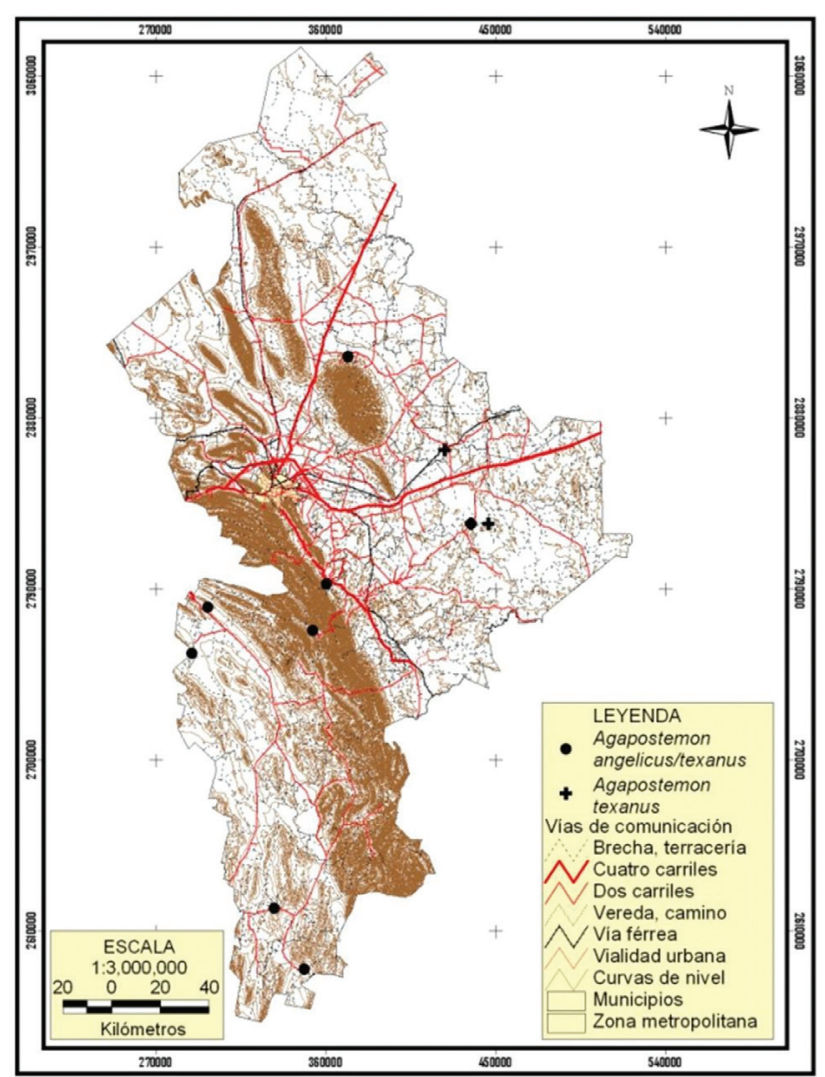

Figura 3. Distribución de Agapostemon angelicus/A. texanus (ㅇ) y A. texanus ( $\overbrace{}^{\top})$ en el estado de Nuevo León.

\section{Discusión}

En México se han realizado diversos estudios sobre abejas silvestres (Labougle, 1990; Ayala, 1999; Meléndez-Ramírez et al., 2002; Godínez et al., 2004; Contreras-Escareño et al., 2008; Fierros-López, 2008; Golubov et al., 2010; Meneses et al., 2010), interacción planta-polinizador (Raine et al., 2007), conservación (Canto-Aguilar y Parra-Tabla, 2000; Meléndez, 2006) y de taxonomía de algunos géneros de Halictidae (SmithPardo, 2005; Engel et al., 2007), y aunque en sus resultados mencionan especies de Agapostemon, ningún trabajo es específico de este género. Por otra parte, estos trabajos son principalmente del centro y sur del país, mientras que para el estado de Nuevo León, sólo la investigación de Ramírez-Freire (2008) alude a las abejas, si bien, no es ése su propósito principal. Por ello, se hace necesario incrementar y fomentar las investigaciones sobre abejas de los estados del Noreste de México con diferentes enfoques.

Muestra de lo anterior es que inicialmente Sonora se ubicaba en la primera posición en cuanto a número de

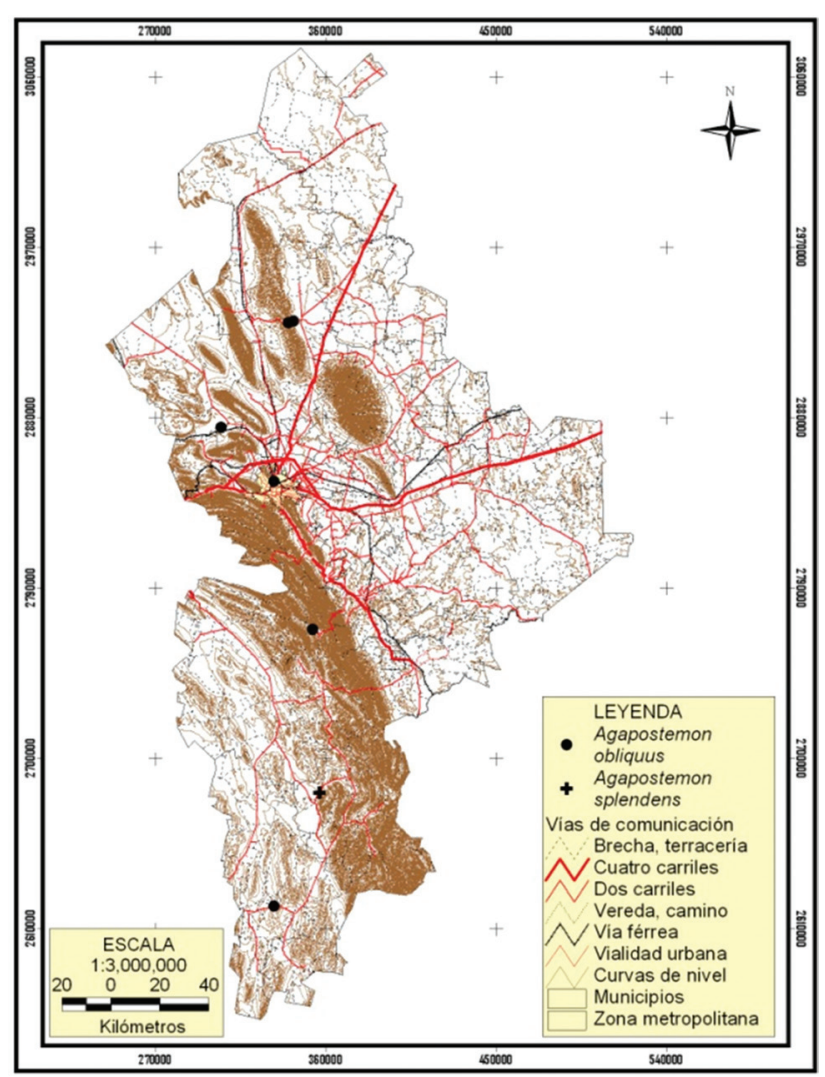

Figura 4. Distribución de Agapostemon obliquus y A. splendens en el estado de Nuevo León.

especies de Agapostemon y Nuevo León en el tercer lugar (Ayala et al., 1996; Ascher y Pickering, 2010; UNIBIO, 2011); ahora Nuevo León escala hasta el primer sitio con 11 especies, lo que hace patente la necesidad de incrementar los trabajos faunísticos en zonas poco exploradas.

Las especies que se registran por primera vez para Nuevo León son taxones que aparecen en colecciones con localidades cercanas a nuestro estado, como A. nasutus, en los estados vecinos de Tamaulipas, San Luis Potosí y Texas (Ascher y Pickering, 2010) y A. splendens, que se cita para Veracruz y Texas (Mitchell, 1960). Agapostemon angelicus es una especie ampliamente distribuida en los Estados Unidos; la localidad de San Ignacio, Texas es la ubicación más cercana a nuestro estado, y en México se registra para Baja California, Sonora, Chihuahua y Durango (Ascher y Pickering, 2010), y Roberts (1972) señala que las hembras se distribuyen en gran parte del territorio nacional. Si no había sido registrada en nuestro estado (al igual que los taxones anteriores), muy probablemente se deba a que éste es el primer estudio formal al respecto y por tanto, podría esperarse que esta especie se hallara también en Nuevo León; de ahí que se haya 


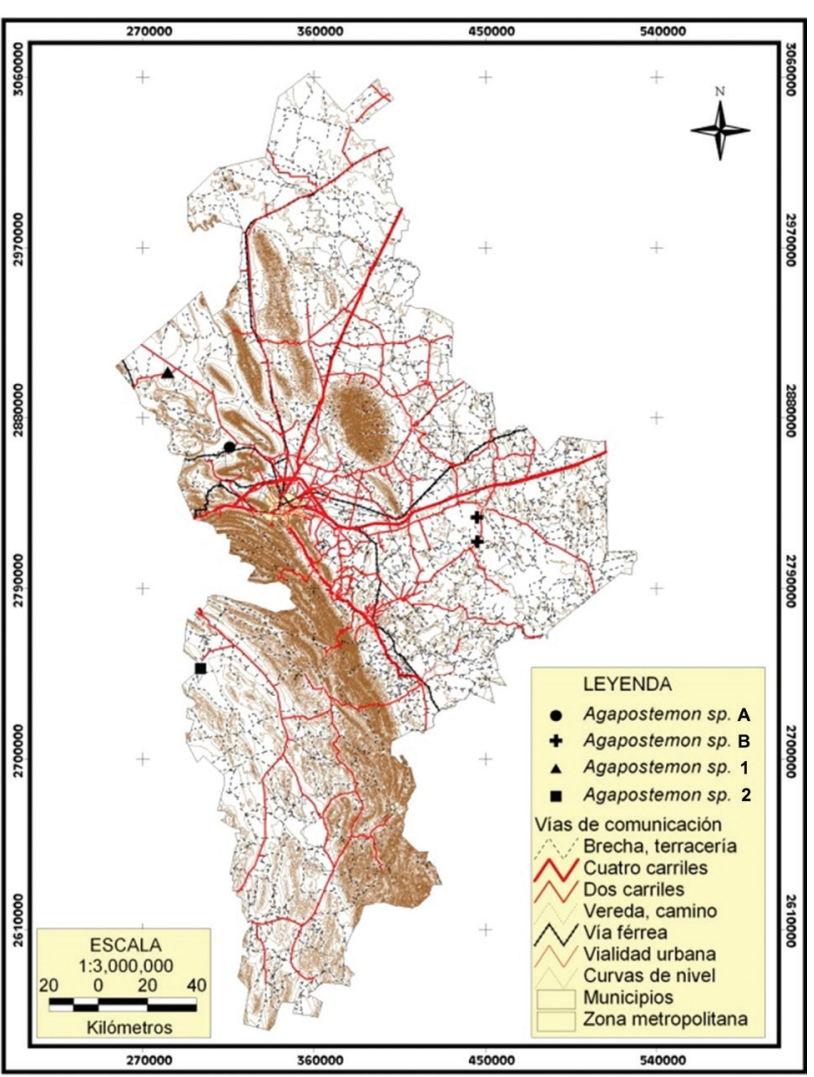

Figura 5. Distribución de Agapostemon sp. A, Agapostemon sp. B, Agapostemon sp. 1 y Agapostemon sp. 2 en el estado de Nuevo León.

denominado A. angelicus / texanus, mientras no se pueda diferenciarla de $A$. texanus.

Respecto a las plantas visitadas por las especies de Agapostemon, se encontró que autores como Hurd y Linsley (1975) y Hurd et al. (1980) lo relacionan mayormente con cucurbitáceas y asteráceas, principalmente del género Helianthus; sin embargo, aquí se encontraron nuevas especies de plantas pertenecientes a otras familias que son fuente de recursos para el Agapostemon, como en el caso de A. angelicus / texanus que se recolectó en Heretia anacua (Boraginaceae); A. melliventris sobre Leucophyllum frutescens (Scrophulariaceae) y Parthenium argentatum (Compositae); A. nasutus en Ludwigia octovalis (Onagraceae); A. obliquus en Eysenhardtia texana (Fabaceae) y Leucophyllum frutescens (Scrophulariaceae), y A. tyleri sobre flores de Opuntia (Cactaceae).

En lo que se refiere al método de captura, es de notarse que los resultados de este trabajo coinciden con los de Campbell y Hanula (2007), Wilson et al. (2008) y Grundel et al. (2011), quienes recolectaron mayor número

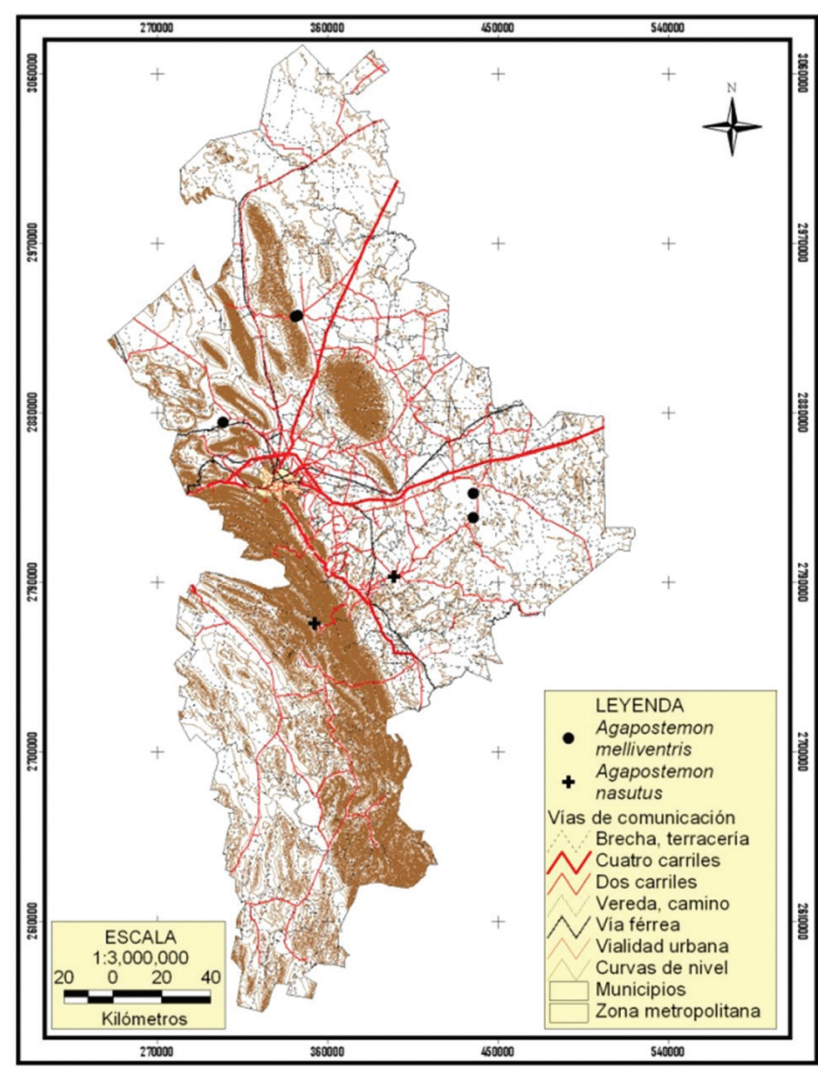

Figura 6. Distribución de Agapostemon melliventris y A. nasutus en el estado de Nuevo León.

de especies en platos trampa (pero en nivel de familia, Halicidae) que mediante el uso de red entomológica. Se observa que el amarillo ha sido el color más utilizado para los platos trampa porque atrae una gran diversidad de insectos (Leong y Thorp, 1999). No obstante, en algunos estudios realizados con platos trampa de éste y otros colores, como en el de Campbell y Hanula (2007), el mayor número de ejemplares se obtuvo con trampas de color azul. Los autores adjudican este resultado a que en las zonas de muestreo no hay muchas plantas de color amarillo, por lo tanto fue menos efectivo. Otros autores, como Wilson et al. (2008) y Grundel et al. (2011), registraron una preferencia de las abejas por el blanco. Sin embargo, en el presente estudio los resultados arrojaron preferencia por el amarillo, color que coincide con el predominante en la flora de nuestro estado, lo que indica que hay que considerar esta característica para lograr mejores resultados en la obtención de un mayor número de especies.

Los tipos de vegetación donde se recolectaron la mayor parte de las abejas fueron los diferentes matorra- 
les, que son la vegetación dominante en el estado (51\%), como lo refiere el INEGI (2005) y fue en éstos donde se encontró la mayor riqueza de especies ( 8 de 11), al igual que en la vegetación de disturbio que, si bien, no se tiene un estimado de la proporción que abarca, el hecho de que en ocasiones no se pueda acceder a ciertas zonas por las características propias de la vegetación o porque son propiedad privada contribuyó en gran medida a que algunos de los muestreos se realizaran a orillas de caminos o en las afueras de las cabeceras municipales, donde el componente principal son plantas de la familia Compositae. Éstas atraen gran número de polinizadores, lo que se explica por la gran riqueza y abundancia de las especies de esta familia. Su amplia distribución, el tipo de inflorescencias (Godinez-García et al., 2004) y el hecho de que se mantengan abiertas todo el día y produzcan abundante cantidad de néctar y polen proporciona un ambiente continuamente favorable para las abejas (Hurd et al., 1980).

En el estado de Nuevo León, las abejas nativas han sido poco estudiadas y su representación en las colecciones entomológicas mexicanas es muy precaria. Curiosamente, existen más ejemplares en colecciones extranjeras, por lo que el presente estudio pretende subsanar esta ausencia de información.

\section{Agradecimientos}

A John L. Neff del Central Texas Melittological Institute por la información facilitada y su asesoría con la identificación de algunas de las especies.

\section{Literatura citada}

Alanís-Flores, G. J., G. Cano y M. Rovalo M. 1996. Vegetación y flora de Nuevo León, una guía botánico-ecológica. Impresora Monterrey, Nuevo León. p. 1-9.

Ascher, J. y J. Pickering. 2010. Discover life's bee species guide. www.discoverlife.org/mp/20q?search=Agapostemon; última consulta 18.III.2010.

Ayala, R. 1999. Revisión de las abejas sin aguijón de México (Hymenoptera: Apidae: Meliponini). Folia Entomológica Mexicana 106:1-123.

Ayala, R., T. L. Griswold y D. Yanega. 1996. Apoidea (Hymenoptera). In Biodiversidad y biogeografía de artrópodos de México. Hacia una síntesis de su conocimiento, J. Llorente B., A. N. García y E. González (eds.). UNAM/CONABIO, México, D. F. p. 423-464.

Ayala, R., T. L. Griswold y S. H. Bullock. 1998. Las abejas nativas de México. In Diversidad biológica de México: orígenes y distribución, T. P. Ramamoorthy, R. Bye, A. Lot y J. Fa (comps.). Instituto de Biología, UNAM, México, D. F. p. 179225.
Buchmann, S. L. y G. P. Nabhan. 1996. The forgotten pollinators. Island, Washington, D.C. 292 p.

Campbell, J. W. y J. L. Hanula. 2007. Efficiency of Malaise traps and colored pan traps for collecting flower visiting insects from three forested ecosystems. Journal of Insect Conservation 11:399-408.

Canto-Aguilar, M. A. y V. Parra-Tabla. 2000. Importance of conserving alternative pollinators: assessing the pollination efficiency of the squash bee, Peponapis limitaris in Cucurbita muschata (Cucurbitaceae). Journal of Insect Conservation 4:203-210.

Contreras-Escareño, F., C. Echazarreta G., M. Vázquez G., J. A. Vázquez G. y F. Becerra-Guzmán. 2008. Diversidad de abejas sin aguijón en dos comunidades de la Sierra de Manantlán Jalisco, México. Revista Apitec 69:4-11.

Dafni, A. y C. O'Toole. 1994. Pollination syndromes in the Mediterranean: Generalisations and peculiarities. In Plantanimal interactions in Mediterranean-type ecosystems, M. Arianoutsou y R. H. Groves (eds.). Kluwer Academic, Dordrecht, Netherlands. p. 125-135.

Daily, G. C. (ed). 1997. Nature's services: societal dependence on natural ecosystems. Island, Washington, D.C. 392 p.

Dias, B. S. F., A. Raw y V. L. Imperatriz-Fonseca. 1999. International pollinators initiative: The Sao Paulo declaration of pollinators. Report on the recommendations of the workshop on the conservation and sustainable use of pollinators in agriculture with emphasis on bee. Brazilian Ministry of the Environment, Brasilia. 79 p.

Droege, S. (comp). 2008. The very handy manual: how to catch and identify bees and manage a collection.www.nbii.gov/ images/uploaded/152986_1215796993080_Handy_Bee_ Manual_Jun_2008.pdf; última consulta: 2.IV.2009.

Engel, M. S., I. A. Hinojosa D. y O. Yáñez O. 2007. The Augochlora like Dialictus from Guatemala and Southern México (Hymenoptera: Halictidae). Acta Zoológica Mexicana (nueva serie) 23:125-134.

Fierros-López, H. E. 2008. Estudio de ordenamiento ecológico territorial de Jalisco, diagnóstico de los subsistemas. www. acude.udg.mx/jalisciencia/diagnostico/biotico/faunajalisco/ abejas/anexo/htm; última consulta: 7.X.2008.

Godínez-García, L. M., I. Hinojosa-Díaz y O. Yáñez-Ordóñez. 2004. Melitofauna (Insecta: Hymenoptera) de algunos bosques mesófilos de montaña. In Biodiversidad de la sierra Madre Oriental, I. Luna, J. J. Morrone y D. Espinosa (eds.). Las Prensas de Ciencias, México, D. F. p. 321-337.

Golubov, J., M. C. Mandujano, A. J. Martínez y J. López-Portillo. 2010. Bee diversity on nectarful and nectarless honey mesquites. Journal of Insect Conservation 14:217-226.

Grundel R., K. J. Frohnapple, R. P. Jean y N. B. Pavlovic. 2011. Effectiveness of bowl trapping and netting for inventory of a bee community. Environmental Entomology 40:374-380.

Hurd, P. D. y E. G. Linsley. 1975. The Principal Larrea Bees of 
the southwestern United States (Hymenoptera: Apoidea). Smithsonian Contributions to Zoology 193:1-74.

Hurd, P. D., W. E. LaBerge y E. G. Linsley. 1980. Principal Sunflower Bees of North America with Emphasis on the Soutwestern United States (Hymenoptera: Apoidea). Smithsonian Institution Press, Washington, D.C. 158 p.

INEGI, 1986. Síntesis geográfica del estado de Nuevo León. Secretaría de Programación y Presupuesto, México, D. F.

INEGI. 2005. Información nacional por entidad y municipio.Medio ambiente. http://www.inegi.org.mx/sistemas/mexicocifras/ default.aspx?ent=19; última consulta: 16.III.2010.

Janjic, J. y L. Packer. 2003. Phylogeny of the bee genus Agapostemon (Hymenoptera: Halictidae). Systematic Entomology 28:101-124.

Labougle, J. M. 1990. Bombus de México y América Central (Hymenoptera: Apidae). Science Bulletin, The University of Kansas 54:35-73.

Leong, J. M. y R. W. Thorp. 1999. Colour-coded sampling: The pan colour preferences of oligolectic and nonoligolectic bees associated with a vernal pool plant. Ecological Entomology 24:329-335

Meléndez-Ramírez, V., S. Magaña-Rueda, V. Parra-Tabla, R. Ayala y J. Navarro. 2002. Diversity of native bees visitors of cucurbit crops (Cucurbitaceae) in Yucatán, Mexico. Journal of Insect Conservation 6:135-147.

Meléndez, R. V. 2006. Conservación de abejas y polinización de cultivos en Yucatán México. Segundas Jornadas de Polinización en Plantas Hortícolas, 19-21 abril 2005, Centro de Investigación y Formación Agraria (CIFA), La Mojonera, Almería. p. 236-244.

Meneses, C. L., V. Meléndez, V. Parra-Tabla y J. Navarro. 2010. Bee diversity in a fragmented landscape of the Mexican neotropic. Journal of Insect Conservation 1:323-334.

Michener, C. D, R. J. McGlinley y B. Danforth. 1994. The bee genera of North and Central America(Hymenoptera:Apoidea). Smithsonian Institution Press, Washington. D.C. 9 p.

Michener, C. D. 2000. The bees of the world. Johns Hopkins University Press, Baltimore, Maryland. 913 p.

Mitchell, T. B. 1960. Bees of the Eastern United States. I. Technical Bulletin (North Carolina Agricultural Experiment
Station) 141. p. 1-538. [Introduction, Andrenidae, Colletidae, Halictidae, Mellitidae].http://insectmuseum.org./ easternBees.php.; última consulta: 31.III. 2009

O'Toole, C. 1993. Diversity of native bees and agroecosystems. In Hymenoptera and biodiversity. Third Quadrennial Symposium of the International Society of Hymenopterists, 1995, J. La Salle y I. Gauld (eds.). London. CABI, Wallingford p. 69-106.

Smith-Pardo, A. 2005. The bees of the genus Neocorynura of México (Hymenoptera: Halictidae: Augochlorini). Folia Entomológica Mexicana 44:165-193.

Raine, N. E., A. Sharp P. y G. N. Stone. 2007. Plant-pollinator interactions in a Mexican Acacia community. ArthropodPlant Interactions 1:101-117.

Ramírez-Freire, L. 2008. La floración de cinco especies de cactáceas y sus insectos asociados en el Área Natural Protegida "Sierra Corral de los Bandidos" municipio de García, Nuevo León. Tesis, Maestría Facultad de Ciencias Biológicas, Universidad Autónoma de Nuevo León, San Nicolás de los Garza, Monterrey. 135 p.

Roberts, R. B. 1972. Revision of the bee genus Agapostemon (Hymenoptera: Halictidae). The University of Kansas Science Bulletin 49:437-590.

Treviño-Garza, E. J., C. Cavazos-Camacho y O. A. AguirreCalderón. 2001. Distribución y estructura de los bosques de galería en dos ríos del centro sur de Nuevo León. Madera y Bosques 7:13-25.

Wilson, J. S., T. Griswold y O. Messinger. 2008. Sampling bee communities (Hymenoptera: Apiformes) in a desert landscape: Are pan traps sufficient? Journal of the Kansas Entomological Society 81:288-300.

UNIBIO. 2011. CNIN/Abejas de México/Apodea. In Unidad de Informática para la Biodiversidad, Instituto de Biología, UNAM, México, D. F. http://unibio.unam.mx/minidigir/ main.jsp?accion $=$ sc\&colecciones $=\mathrm{CNIN}$; última consulta: 02.II.2011.

Yáñez-Ordóñez, O. e I. Hinojosa-Díaz. 2004. La Colección Himenopterológica (Insecta) del Museo de Zoología "Alfonso L. Herrera" de la Facultad de Ciencias, UNAM, México. Acta Zoológica Mexicana 20:167-197. 
Apéndice 1. Especies recolectadas.

\section{Agapostemon angelicus /A. texanus}

NUEVO LEÓN. Agualeguas: 41 q, ejido Rancho Nuevo, 407965E 2912229N, 345 m, 14 III 2010, en platos azul (7), blanco (5) y amarillo (29), matorral espinoso tamaulipeco. Allende: 1 q, camino Raíces-La Trinidad, 396609E 2792757N, 488 m, 22 III 2009, en Ehretia anacua, Vegetación de Disturbio. China: 2 +, carretera China-Terán, 473095E 2824306N, 207 m, 27 VI 2009, en Helianthus annuus, vegetación de disturbio (ruderal). Dr. Arroyo: 1 ㅇ, carretera a Matehuala, 368786E 2622056N, 1956 m, 15 XI 2009 , en plato amarillo, matorral desértico micrófilo. Galeana: 59 오, Llano de la Soledad, 325417E 2756154N, 1885 m, 10 IV 2010 , en platos azul (8), blanco (18) y amarillo (3), matorral de gobernadora (Larrea tridentata); 1 ㅇ, camino a ejido El Pinal 336070E 2785806N, 2173 m, 1 V 2010, en plato amarillo, ecotono de matorral desértico y bosque de pino (Pinus cembroides). Mier y Noriega: 3 q, $384675 \mathrm{E}$ 2590154N, 1641 m, 14 XI 2009, en platos blancos (2) y amarillo (1), matorral desértico micrófilo. Rayones: 3 +, carretera RayonesCasillas a $2.7 \mathrm{~km}$ de cabecera municipal, 389265E 2768265N, $887 \mathrm{~m}, 16 \mathrm{~V} 2009$, en plato blanco (1) y amarillo (2), vegetación de disturbio (huerto de nogal de Carya illinoinensis).

Agapostemon leunculus Vachal, 1903

En el presente estudio no se obtuvieron ejemplares de esta especie; sin embargo se informó que fue recolectado en 1976 en la localidad de Chipinque (Ascher y Pickering, 2010).

Agapostemon melliventris Cresson, 1874

Agapostemon fasciatus Crawford, 1901

Halictus (Agapostemon) plurifasciatus Vachal, 1903

Agapostemon digueti Cockerell, 1924

NUEVO LEÓN. China: 2 9, Parque Estatal El Cuchillo, 472967E 2837230N, 205 m, 27 VI 2009, en plato azul y blanco, vegetación de disturbio; 2 9, carretera China-Terán, 473095E 2824306N, 207 m, 27 VI 2009, en Helianthus annuus y Partenium argentatum, vegetación de disturbio (ruderal). Mina: 1 +, ex hacienda del Muerto, 340909E 2874933N, 632 m, 25 VII 2009, en plato blanco, matorral bajo subinerme. Sabinas Hidalgo, 8 ㅇ, 379147E 2930982N, 370 m, 12 IX 2009, en Leucophyllum frutescens, matorral submontano; Avenida Principal, 1 q,380449 E 2931453 N, 317 m, 12 IX 2009, en Leucophyllum frutescens.

Agapostemon nasutus Smith, 1853

NUEVO LEÓN. Rayones: 1 +, carretera Rayones-Casillas, 389265E 2768265N, 887 m, 16 V 2009, en plato amarillo, vegetación de disturbio (huerto de nogal de Carya illinoinensis). Gral. Terán: 1 J , a orillas del río Pilón, 431092E 2793197N, 335 m, 27 VI 2009, en Ludwigia octovalis, vegetación riparia.

Agapostemon obliquus (Provancher, 1888)

Augochlora obliqua Provancher, 1888

Agapostemon cockerelli Crawford, 1901

NUEVO LEÓN. Mina: 1 †, ex hacienda del Muerto, 630 m, 21 XII 2008, en Eysenhardtia texana, matorral desértico micrófilo. Rayones: 1 q, carretera Rayones-Casillas, 389265E 2768265N, 887 m, 16 V 2009, en plato azul, vegetación de disturbio (huerto de nogal de Carya illinoinensis). San Nicolás de los Garza: 2 o, Cd. Universitaria, 368838 E 2846320 N, 520 m, en Leucophyllum frutescens y plato azul, zona de disturbio (jardín desértico en área urbana). Sabinas Hidalgo, 2 + $, 379147 \mathrm{E} 2930982 \mathrm{~N}, 324$ m, en Leucophyllum frutescens, vegetación de disturbio (ruderal); 4 을 carretera Sabinas-Villaldama, 376436E 2930246N, 336 m, plato amarillo (3) y azul (1), vegetación de disturbio. Dr. Arroyo: 1 P, carretera a Matehuala, 368786E 2622056N, 1956 m, 15 XI 2009, en plato amarillo, matorral desértico micrófilo.

Agapostemon splendens (Lepeletier, 1841)

Halictus splendens Lepeletier, 1841

Agapostemon aeruginosus Smith, 1853

NUEVO LEÓN. Aramberri: 1 ô, camino a Sandia, 393020 E 2681970N, 1758 m, 19 VIII 2009, en Compositae, vegetación de disturbio (ruderal).

Agapostemon texanus Cresson, 1872

Agapostemon texanus subtilior Cockerell, 1898

Agapostemon borealis Crawford, 1901

Agapostemon californicus Crawford, 1901

Halictus (Agapostemon) brachycerus Vachal, 1903

Agapostemon texanus iowensis Cockerell, 1910

Agapostemon proscriptus Cockerell, 1912

Agapostemon joseanus Friese, 1917 [“1916“] 
Apéndice 1. Continúa.

Agapostemon sulfuripes Friese, 1917 [“1916“]

Agapostemon cyanozonus Cockerell, 1924

Agapostemon proscriptellus Cockerell, 1924

Agapostemon texanus vandykei Cockerell, 1925

Agapostemon californicus psammobius Cockerell, 1937

Agapostemon angelicus idahoensis Michener, 1937

Agapostemon californicus clementinus Cockerell, 1939

NUEVO LEÓN. China: 1 స̂, carretera China-Terán, 473095E 2824306, 207 m, 27 VI 2009, en Helianthus annuus, vegetación de disturbio (ruderal). Los Herrera: 1 ô, brecha a Rancho San José, 4509102E 2863255 N, 201 m, 28 VI 2009, en plato amarillo, matorral mediano espinoso.

Agapostemon tyleri Cockerell, 1917

NUEVO LEÓN. China: 4 †, 472967E 2837230N, 205 m, 27 VI 2009, en plato amarillo (1), azul (2) y blanco (1), vegetación de disturbio. Dr. Arroyo: 2 Oे, 368787E y 2622056N, 1956 m, 15 XI 2009, en plato amarillo, matorral desértico micrófilo. Galeana: 37 o, Llano de la Soledad, 325417E y 2756154N, 1885 m, 10 IV 2010, en plato amarillo (17), azul (9) y blanco (11), matorral de gobernadora (Larrea tridentata); 4 + , camino al Pinal, 333728E 2780526N, 2173 m, 1 V 2010, en plato azul (1) y blanco (3), ecotono de matorral desértico y bosque de pino (Pinus cembroides); 3 \&, camino a ejido 18 de Marzo, 400517E 2731711N, 1853 m, 16 IV 2009 , en Opuntia sp., bosque de pino (P. pseudostrobus). García: 15 \&, ANP sierra Corral de los Bandidos, 324858E 2838204N, 1250 m, 11 VII 2009 , en plato amarillo (8) y blanco (7), matorral desértico micrófilo. Los Herrera: 1 đ̃, brecha a Rancho San José, 459102E 2863255N, 201 m, 28 VI 2009, en plato blanco, matorral mediano espinoso. Mier y Noriega: 4 q, 384675E 2590154N, 1641 m, 14 XI 2009 , en plato azul (2) y blanco (2), matorral desértico micrófilo. Mina, 1 đ̃, camino al Espinazo, 307713E 2914833N, 703 m, 4 X 2009, plato amarillo, matorral bajo subinerme. Rayones: 4 \%, carretera Galeana-Rayones, 392043E 2756018N, 1436 m, 17 IV 2009 , en Opuntia microdasys, matorral submontano; 2 ㅇ, carretera de Rayones-Casillas, 389265E 2768265N, 887 m, 16 V 2009, en plato amarillo, vegetación disturbio (huerto de nogal de Carya illinoinensis). Santa Catarina: 8 + , cañón de la Huasteca, 360506E 2828103N, 844 m, 3 IV 2010, en plato amarillo (7) y blanco (1), matorral desértico micrófilo.

Agapostemon sp. A

NUEVO LEÓN. Mina: 1 đ̊, 340909E y 2874933N, 632 m, 25 VII 2009, en plato amarillo, matorral bajo subinerme.

Agapostemon sp. B

NUEVO LEÓN. China: 1 §ૈ, El Cuchillo, 472967E 2837230N, 205 m, 27 VI 2009, en plato amarillo (1), vegetación de disturbio; 1 đૈ, carretera China-Terán, 473095E y 2824306N, 207 m, 27 VI 2009, en Helianthus annuus, vegetación de disturbio,

\section{Agapostemon sp. 1}

NUEVO LEÓN. Mina: 6 ㅇ, camino al Espinazo, 307714 E y 2914833 N, 703 m, 4 X 2009, en plato amarillo (2), blanco (1) y azul (3), matorral bajo subinerme.

\section{Agapostemon sp. 2}

NUEVO LEÓN. Galeana: 1 ㅇ, Llano de la Soledad, 325417 E y 2756154 N, 1885 m, 10 IV 2009, en plato amarillo, matorral de gobernadora (Larrea tridentata). 